\title{
Effect of Transcutaneous Electrical Nerve Stimulation Versus Transcranial Direct Current Stimulation on Diabetic Peripheral Neuropathy
}

\author{
AWNY F. RAHMY, Ph.D.; SHEREEN H. EL-SAID, Ph.D. and ASMAA YACOUB, M.Sc. \\ The Department of Cardiovascular/Respiratory Disorder and Geriatrics, Faculty of Physical Therapy, Cairo University
}

\begin{abstract}
Background: Diabetic periphral neuropathy is probably the most common form of the diabetic neuropathies. It is seen in both type 1 and type $2 \mathrm{DM}$ with similar frequency, and may occur at time of diagnosis of type $2 \mathrm{DM}$. Sensory symptoms are more prominent than motor symptoms and usually involve the lower limbs.
\end{abstract}

Objective: To compare the effect of transcutaneous electrical nerve stimulation versus transcranial direct current stimulation on diabetic painful neuropathy.

Subjects and Methods: Forty patients of both sex suffering from diabetic peripheral neuropathy with age range from 50 to 60 years old were participated in this study. They were assigned randomly into two equal groups, Group (A) received transcranial direct current stimulation, Group (B) received transcutaneous electrical nerve stimulation. Treatment was applied 3 times per week for 2 months. Pain intensity was assessed by Neuropathy Pain Scale (NPS) before and after treatment.

Results: There was significant improvement in both groups after treatment than before treatment but there was no significant difference between transcranial direct current stimulation and transcutaneous electrical nerve stimulation.

Conclusion: Both transcutaneous electrical nerve stimulation and transcranial direct current stimulation are both effective in pain associated with diabetic painful neuropathy.

Key Words: Transcranial direct current stimulation - Transcutaneous electrical nerve stimulation - Diabetic peripheral neuropathy.

\section{Introduction}

DIABETIC Peripheral Neuropathy (DPN) is the most common complication of diabetes. It results in a plenty of syndromes so there is no universally accepted classification. They can be subdivided

Correspondence to: Dr. Awny F. Rahmy, The Department of Cardiovascular/Respiratory Disorder and Geriatrics, Faculty of Physical Therapy, Cairo University into focal or multifocal neuropathies, including diabetic amyotrophy, symmetric polyneuropathies, including sensorimotor polyneuropathy (DSPN) which is the most common type, affecting about $30 \%$ of diabetic patients admitted to hospital and $25 \%$ of those in around community [1] .

\section{Distal sensory diabetic polyneuropathy:}

It is the most common form of neuropathy in diabetes, about $50 \%$ of patients may experience a lot of symptoms as burning pain, electrical or stabbing sensations, paraesthesia, hyperaesthesia, and deep aching pain [2]. These symptoms become worse at night and make patient awake from sleep. Together with painful symptoms during the day causes a reduction in the individual's ability to perform daily activities and affect his quality of life [3].

Diabetic periphral neuropathy is probably the most common form of the diabetic neuropathies. It is seen in both type 1 and type $2 \mathrm{DM}$ with similar frequency, and may be present at the time of diagnosis of type $2 \mathrm{DM}$. Sensory symptoms occur more than motor one and usually affect the lower limbs as pain, paresthesias, hyperesthesias, deep aching, burning, and sharp stabbing sensations similar those occur in acute sensory neuropathy but less severe than them. In addition, patients may suffer from negative symptoms such as numbness in the feet and legs, leading i to painless foot ulcers and subsequent amputations if the neuropathy is not rapidly recognized and treated. Precarious is also frequently seen, owing to abnormal proprioception and muscle sensory function. Alternatively, some patients may have no symptoms, or signs and neuropathy only appear by a detailed neurologic examination. On physical examination a symmetric stocking-like distribution of sensory abnormalities 
in both lower limbs is usually seen. In more severe cases the hands may be involved [4]. The origin of pain in periphral diabetic neuropathy is not fully understood. The abnormalities in the peripheral or central nervous system could be attributed to hyperglycaemia, as this is the key metabolic abnormality of diabetes $[\mathbf{r}$.

Electrotherapy could be effective in case of impaired microcirculation that occur in the peripheral nerves of patients with diabetic poly neuropathy. An increase in the muscle's oxidative capacity (metabolic effect of electrotherapy) is also important. Electrical stimulation produce an effect on the morphological and metabolic properties of paralysed muscles leading to local release of neurotransmitters such as serotonin which increased the production of mitochondrial Adenosine Triphosphate (ATP), release of endorphins, also antiinflammatory effects may enhance the analgesic effect of electrotherapy. Activation of the dorsal column occur as a further potential mechanism of the effect of electrotherapy. The input of pain is interrupted by the inhibition of $\mathrm{C}$ fibers (thus interrupting/gating the input of pain). There is reduction in the excitability of the human motor cortex by the use of high frequency Transcutaneous Electrical Nerve Stimulation (TENS) [6]. Neuropathic pain is associated with a functional reorganization and hyperexitability of the somatosensory and motor cortex. So it is beneficial to reverse cortical reorganization in patients with spontaneous or provoked pain as this will result in pain relief. Brain stimulation techniques such as transcranial Direct Current Stimulation (tDCS) was found to be suitable methods for modulation of cortical excitability. In addition, tDCS is easy to apply, portable and not expensive, which enhances the clinical potential of this technique [7], a structured review of different types of electrotherapy and their effect on painful diabetic peripheral neuropathy was therefore performed.

\section{Material and Methods}

Forty patients from both sex with diabetic painful neuropathy participated in this randomized controlled study, their age ranged from 50-60 years. They were chosen from diabetes out clinic. (Kasr El-Eini Hospital) this study is conducted from January 2014 till July 2016. Patients were randomly assigned into two equal groups in numbers, twenty patients in each group:

Group A were treated Transcranial Direct Current Stimulation (tDCS) with 20min, 3 sessions/ week for 2 successive months.
Group B were treated with Transcutaneous Electrical Nerve Stimulation (TENS) 20min, 3 sessions/week for 2 successive months.

Both groups have the same assessment and the same medical treatment.

\section{Inclusion criteria:}

- All patients suffer from: diabetes $>5$ years ago.

- Mean pain levels $\geq 4$ out 10 on VAS.

- Patients' age ranged from 50 to 60 years.

Exclusion criteria:

- Patients with unstable medical or psychiatric disorder.

- Patients with implanted devices for pain control such as vagal or deep brain stimulator.

- Bedridden patients.

- Pain not attributed to other causes such as spinal cord injury.

\section{Instrumentation:}

For assessment of pain:

Neuropathy Pain Scale (NPS) to determine the pain severity before and after treatment.

\section{For treatment:}

- Transcutaneous Electrical Nerve Stimulation (TENS).

The Phyaction Guidance E Pasweg 6A 3740 Bilzen Belgium with power supply 85 VA max is an advanced 2 channel appliance for electrotherapy, offering a wide range of current types which can be applied by means of normal electrodes or via the integrated vacuum unit (Article Number 323254).

Procedures of application of transcutaneous electrical nerve stimulation:

Before starting the treatment, the treatment procedures was explained for the patients.

\section{Settings of the apparatus and steps of stimulation:}

- The electrodes are placed on or around the painful area and at least one pad width apart.

- Intensity increased slowly (by turning the dials) until patient feel a 'strong but comfortable' tingling sensation according to patient tolerance. Transcutaneos electrical nerve stimulation was applied for 20 minutes per session, 3 times per week. 
Transcranial direct current stimulation (Uniphy Phyaction 787).

Gymna-uniphy (phy-action 787) is an advanced Electro-therapeutic appliance. It is able to offer every form of electro-therapy from low frequency to medium frequency currents passing through one or two output channels, with or without making use of the build-in vacuum unit. (Service information Phyaction 787).

Procedures of application of transcranial direct current stimulation:

Before starting the treatment, the treatment procedures was explained for the patients. lation:

- Settings of the apparatus and steps of stimu-

- The patient was relaxed, comfortable and awake during the procedure.

- DC increased current gradually over several seconds until reaching $1 \mathrm{~mA}$ (current density of $0.04 \mathrm{~mA} / \mathrm{cm}^{2}$ ) tDCS.

- Anode electrode was placed over primary motor cortex while cathode was placed over the supraorbital area.

- At the beginning of stimulation, most patients felt a slight itching sensation, which then disappear. Patient may complain from dizziness or vertigo occasionally reported when current is suddenly increased or decreased 8 .

- Session must be stopped if the patient feels headache, sever itching sensation or sever numbness under the electrode areas, dizziness or sense of vomiting.

- Transcranial direct current stimulation was applied for 20 minutes per session, 3times per week.

\section{Statistical analysis:}

- Descriptive statistics and $t$-test was conducted for comparison of the mean age, weight, height, BMI and screening test score between both groups.

- Chi squared test for comparison of sex distribution between groups.

- $t$-test was conducted for comparison of pre and post treatment mean values of neuropathy pain scale between groups.

- Paired $t$-test for comparison between pre and post treatment mean values of neuropathy pain scale in each group.

- The level of significance for all statistical tests was set at $p<0.05$.
- All statistical measures were performed through the Statistical Package for Social Studies (SPSS) Version 19 for windows.

\section{Results}

The purpose of this study was to investigate the effect of Transcutaneous Electrical Nerve Stimulation (TENS) versus transcranial Direct Current Stimulation (tDCS) on diabetic painful neuropathy.

Forty patients with diabetic painful neuropathy. Participated in the study. Patients were subdivided into two groups; twenty patients in each group. The first group was group (A) who received Transcranial Direct current stimulation (tDCS) the second group was the group (B) who received; Transcutaneous Electrical Nerve Stimulation (TENS).

Data obtained from both groups regarding neuropathy pain scale before initiation and following the treatment program were statistically analyzed and compared.

\section{General characteristics of the subjects:}

\section{Group A:}

Twenty patients were included in this group that received tDCS. Their mean \pm SD age, weight, height, and BMI were $52.8 \pm 1.88$ years, $88.2 \pm$ $3.95 \mathrm{~kg}, 155.1 \pm 3.97 \mathrm{~cm}$, and $36.73 \pm 2.49 \mathrm{~kg} / \mathrm{m}^{2} \mathrm{re}-$ spectively.

\section{Group B:}

Twenty patients were included in this group that received TENS. Their mean \pm SD age, weight, height, and BMI were $53.05 \pm 1.93$ years, $88.15 \pm$ $2.47 \mathrm{~kg}, 156 \pm 2.8 \mathrm{~cm}$, and $36.25 \pm 1.68 \mathrm{~kg} / \mathrm{m}^{2}$ respectively.

Comparing the general characteristics of the subjects of both groups revealed that there was no significance difference between both groups in the mean age, weight, height, or BMI $(p>0.05)$.

\section{Sex distribution:}

The sex distribution in group A revealed that there were 12 females with reported percentage of $60 \%$ and 8 males with reported percentage of $40 \%$. The sex distribution in group $\mathrm{B}$ revealed that there were 14 females with reported percentage of $70 \%$ and 6 males with reported percentage of $30 \%$ as shown in and demonstrated in Fig. (1). There was no significant difference between both groups in sex distribution $(p=0.5)$. 

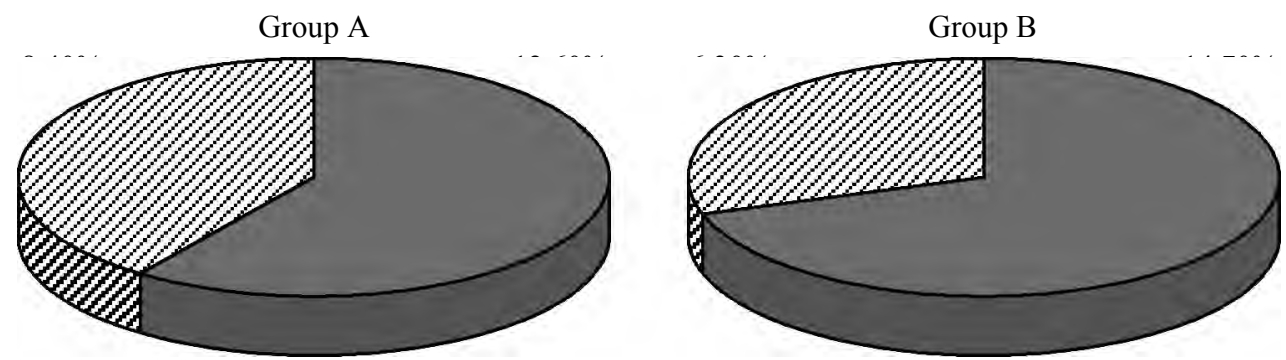

Females

QC Males

Fig. (1): Sex distribution of group A and B.

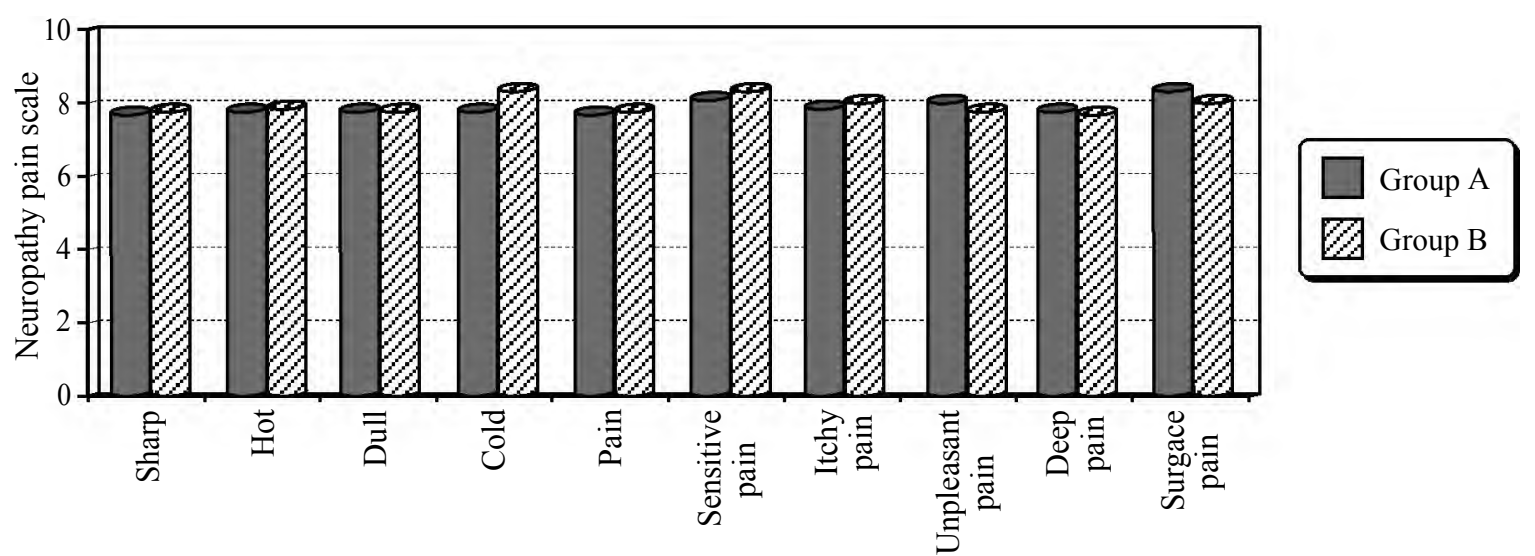

Fig. (2): Pre-treatment mean values of neuropathy pain scale of group A and B.

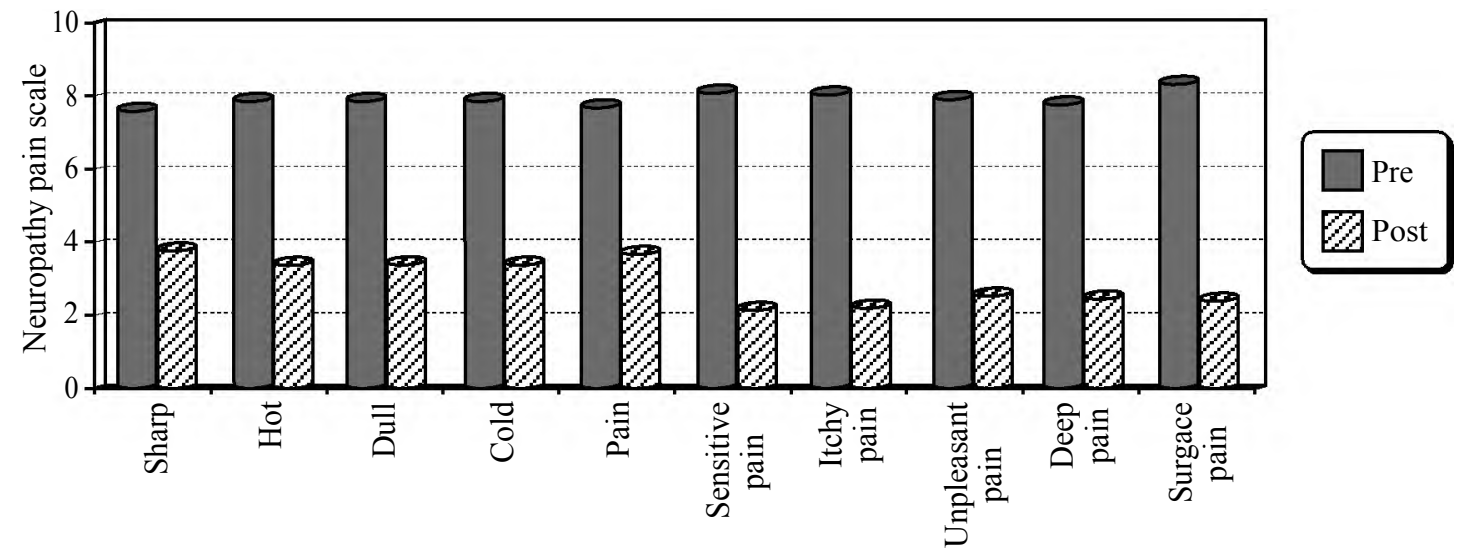

Fig. (3): Pre and post-treatment mean values of neuropathy pain scale of group A.

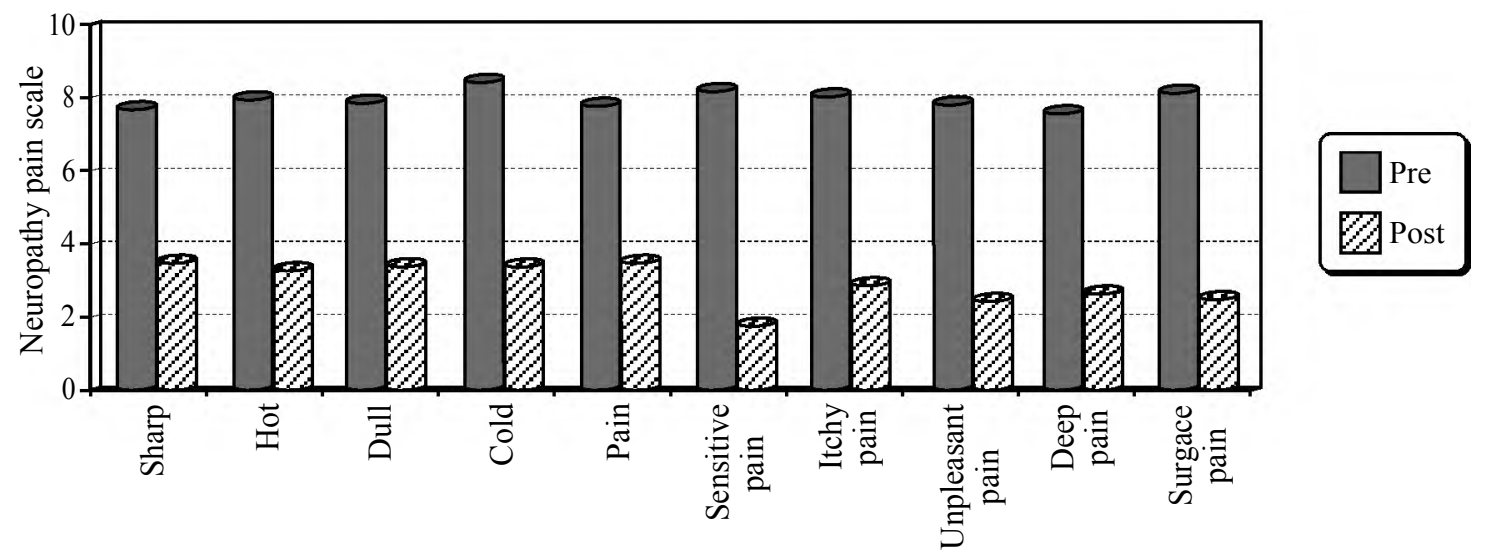

Fig. (4): Pre and post-treatment mean values of neuropathy pain scale of group B. 


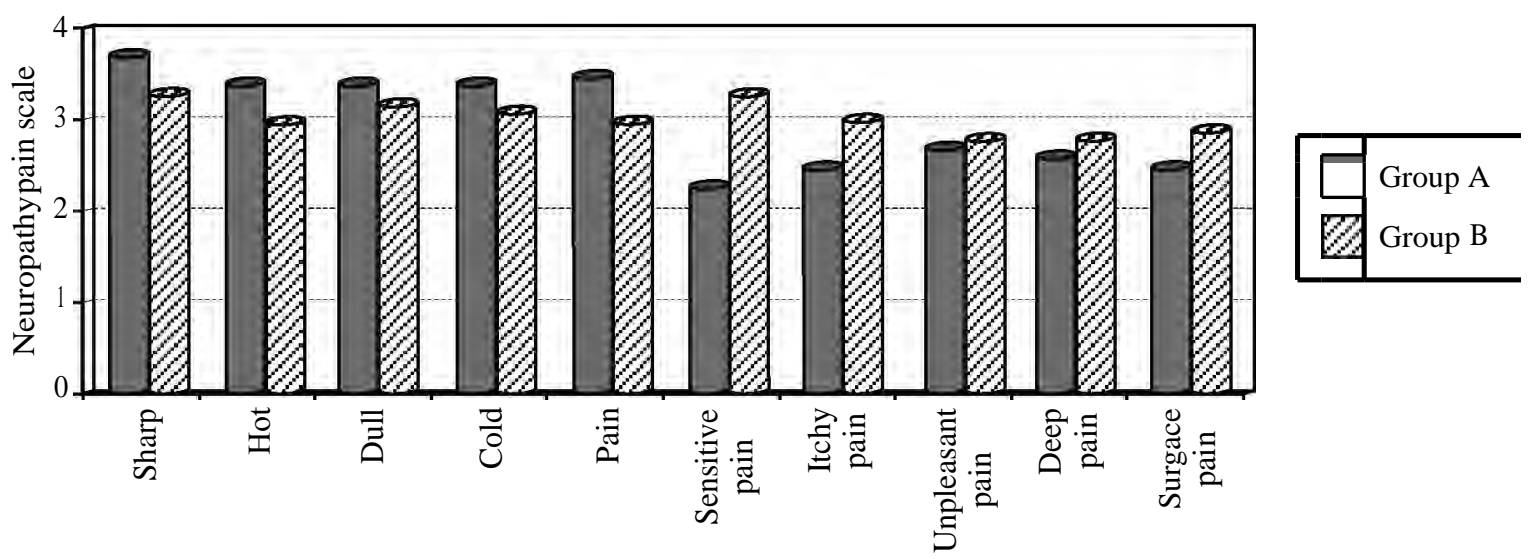

Fig. (5): Post-treatment mean values of neuropathy pain scale of group A and B.

\section{Discussion}

The results of the present study stated that: There is significant improvement in symptomatic pain in both groups after treatment according to neuropathy pain sale scores.

There is no significant difference in improvement statistically between both groups after treatment according to neuropathy pain sale score.

The reduction in pain scores post-treatment program in both groups ( $\mathrm{A}$ and $\mathrm{B}$ ) that occur in this study agreed with Antal et al., [9] who found that the use of $4 \times 4 \mathrm{~cm}$ electrodes in 12 patients with chronic pain syndromes that don't respond to usual therapies. It showed a great reduction on VAS after they have treated with tDCS and continue at day 28 after stimulation. Patients who received sham tDCS after follow-up of patients there was pain reduction in the active group following the first stimulation. The maximal pain reduction occurred after the fifth stimulation. The targeted area of stimulation in the present study was the motor cortex (M1) as it affects GABAergic divisions of thalamus via cortico-thalamic pathways. GABA then inhibits the thalamocortical tracks projecting into the posterior gyrus. It inhibits sensory discriminatory components and the emotional affective ones so stimulating M1 is effective in the treatment of central neuropathic pain.

In another study done by Fregni et al., [10] who used transcranial direct current stimulation over Dorsolateral Prefrontal Cortex (DLPC) to alleviate chronic neuropathic pain.

These results agreed with many other studies that used transcranial direct current stimulation to relief central neuropathic pain due to other causes. Silva et al., [11] suggested that: Anodal stimulation of M1 with direct current may induce analgesic effects in patients with cancer pain. Neuropathy Pain Scale (NPS) values in this study showed a significant improvement in pain between pre and post-treatment in both groups, the percentage of improvement was $53.54 \%$. These agreed with Mori et al., [12] who found significant pain improvement after anodal stimulation with DC over the motor cortex as assessed by VAS for pain and McGill questionnaire, and of overall quality of life.

Results of the present study revealed significant improvement in sharp pain which agreed with Terney et al., [13] who tested the effectiveness of stimulation of M1 with DC of intensity $2 \mathrm{~mA}$ for 5 consecutive days for 20 minutes over the M1 and compared with sham tDCS who found a statistically significant decrease in VAS measure of pain intensity by $37 \%$ in 19 patients with chronic neuropathic pain and persist for 4 weeks after end of treatment.

The application of transcranial direct current stimulation in our study didn't cause any hazards or side effects on the patients. Only at the beginning of stimulation patient felt uncomfortable sensation which disappear in a minute or less of the stimulation. These observations agreed with Minhas et al., [14] who studied the safety of TDCS and noted that as there is no direct contact between the brain and surface electrodes also there is sufficient distance between the electrodes, electrochemical or heating lesions to the neuronal tissue is not common.

The result also agrees with the study done by Soler et al., [15] who proved significant improvement in neuropathic pain at the third month after the combined anodal tDCS with walking Visual Illusion (VI) treatment.

The significant decrease in sharp, sensitive, unpleasant pain that occur according to NPS scores 
agree with Valle et al., [16] who examined differences in results after stimulation with tDCS over the M1 and DLPFC respectively, (delivered for $20 \mathrm{~min}$ at $2 \mathrm{~mA}$ with electrodes $35 \mathrm{~cm}^{2}$ at 10 sessions over 2 weeks) he found that both M1 and DLPFC stimulation resulted in a significant decrease in pain intensity, with over all improvement on quality-of-life measures immediately after the last tDCS session, patients were re-examined after 30-60 days and found that only the M 1 stimulation yielded longer lasting pain relief thorough the 60-day follow-up.

Likewise, another meta-analysis done by Vaseghi et al., [17] supported that anodal stimulation over M1 with-tDCS significantly reduces pain levels by nearly $15 \%$ measured with the Visual Analog Scale (VAS) in patients with chronic pain.

On the other hand analysis of tDCS studies (11 studies, 193 patients) demonstrated significant heterogeneity and did not find a significant difference between active and sham stimulation (very low-quality evidence). Pre-specified sub group analysis of tDCS applied to the motor cortex $(n=183)$ did not demonstrate statistically significant effect and this lack of effect was consistent for subgroups of single or multiple-dose studies [18]

The TENS technique reduces pain through peripheral and central mechanisms. This modality involves stimulation of nerves by applying electrical current to the distribution of nerve fibers via skin surface electrodes. It triggers endogenous opioid release, modifies electrical transmission, and dilates blood vessels, all lead to a reduction in neuropathic pain [19]. The result of this study that stated significant improvement in pain relief after treatment agrees with [20] that reported that application of TENS is safe and effective for treating symptomatic diabetic Periphral neuropathy. This agree with Dubinsky and Miyasaki [21] who compared active TENS with sham-TENS and compared highfrequency muscle stimulation with TENS and found that TENS is effective for reducing pain that arises from diabetic peripheral neuropathy.

In the present study the significant reduction in pain that occurs in group B after application of TENS agrees with meta-analysis of randomized controlled trials conducted by Jin et al., [20] that found that TENS is, it also cause significant decrease in mean pain scores at 4 and 6 weeks of treatment, resulting in improvement in overall neuropathic pain symptoms at 12 weeks.

The results of the present study go on with a systematic review conducted with 8 Nitsche et al., that assessed the effect of different types of electrotherapy including TENS on painful Diabetic Peripheral Neuropathy (DPN) and found significant improvement in pain score after treatment with TENS, he also recommends the use of TENS to achieve improvement in symptomps as long-term therapy and for maintenance of effects in patients with DPN. Also Stein, et al., [22] favors the use of TENS for patients with painful diabetic peripheral neuropathy as TENS significantly improved the symptoms associated with DPN.

During the present study no patients complain of any important adverse effects of TENS treatment, this go on with Donnelan et al., [23] that reported no complain of unpleasant sensations after using TENS to treats patients with sensory impairments after stroke. This agree with Laufer et al., [24] that TENS can easily be applied and tolerated if the stimulations are at a pleasant sensory level in patients with stroke with no adverse effects.

The reduction in all types of pain that statistically occur after application of TENS according to NPS agree with recent study done by Celik et al., [25] who found that low frequency TENS may be most effective for pain in people with spinal cord injury.

TENS effects can be subdivided into analgesic and non-analgesic effects. Clinically TENS is commonly used for its symptomatic relief of pain although there is increasing use of TENS as an antiemetic and for improving of the blood flow in ischemic tissue and wounds. There is less published research on the non-analgesic effects of TENS and some of them is paradoxically, this agree with Norrbrink [26] who investigated the effect of low and high frequency TENS in patients with spinal cord injury, and found that there is no significant improvement in pain intensity ratings, also Oosterhof et al., [27] found that pain intensity was not influenced by TENS or placebo TENS.

\section{Conclusion:}

Both transcranial direct current stimulation and transcutaneous electrical nerve are both effective in relieving pain associated with diabetic painful neuropathy which causes overall improvement in quality of life but there is no significant difference between them.

\section{References}

1- ZIEGLER D., PAPANAS N., VINIK A.I. and SHAW J.E.: Epidemiology of polyneuropathy in diabetes and prediabetes. In Handbook of Clinical Neurology; Diabetes and the Nervous System. 3 rd series ed. Zochodne DW, Malik RA, Eds. Elsevier, p. 3-22, 2014. 
2- DELI G., BOSNYAK E., PUSCH G., KOMOLY S. and FEHER G.: Diabetic Neuropathies: Diagnosis and Management. Neuroendocrinology, Vol. 98, No. 4-Karger Publishers, 2013.

3- TESFAYE S. and SELVARAJAH D.: Advances in the epidemiology, pathogenesis and management of diabetic peripheral neuropathy. Diabetes Metab. Res. Rev., 28 (suppl 1): 8-14 External Resources, 2012.

4- VINIK A.I., MARIE-LAURE N., CASELLINI C. and PARSON H.: Diabetic Neuropathy, Endocrinol. Metab. Clin. N. Am., 42: 747-78. doi.org/10.1016/j.ecl.2013 06.001, 2013.

5- ASLAM A., SINGH J. and RAJBHANDARI: Pain Research and Treatment, Volume 2014, Article ID 412041 , 7 pages. doi.org/10.1155/2014/412041, 2014.

6- PIEBER K.: Electropathy For The Treatment Of Painful Diabetic Periphral Neuropathy J. Rehabil. Med., 42; 28995@ The Authors. doi: 10.2340/16501977-0554, 2010.

7- KNOTKOVA H. and RICARDO A.: Cruciani: Noninvasive Transcranial Direct Current Stimulation for the Study and Treatment of Neuropathic Pain Arpad Szallasi (ed.), Analgesia: Methods and Protocols, Methods in Molecular Biology, Vol. 617, Doi 10.1007/978-1-60327323-7-37.

8- NITSCHE M.A., COHEN L.G., WASSERMANN E.M., PRIORI A. and LANG N.: Transcranial direct current stimulation: State of the art Elsevier. Brain stimulation, 206-23, 2008.

9- ANTAL A., TERNEY D., KÜHNL S. and PAULUS W.: Anodal transcranial direct current stimulation of the motor cortex ameliorates chronic pain and reduces short intracortical inhibition. J. Pain Symptom. Manage., 39: 890903, 2010.

10- FREGNI F., FREEDMAN S. and PASCUAL-LEONE A.: Recent advances in the treatment of chronic pain with non-invasive brain stimulation techniques. Lancet Neuro., 16: 188-91, 2007.

11- SILVA G., MIKSAD R. and FREEDMAN S.: Treatment of cancer pain with noninvasive brain stimulation. J. Pain Symptom Manage., 34: 342-5, 2007.

12- MORI F., CODECÁ C., KUSAYANAGI H., MONTELEONE F., BUTTARI F., FIORE S., et al.: Effects of anodal transcranial direct current stimulation on chronic neuropathic pain in patients with multiple sclerosis. J. Pain, 11: 436-42, 2010.

13- TERNEY D., CHAIEB L., MOLIADZE V., ANTAL A. and PAULUS W.: Increasing human brainexcitability by transcranial high-frequency random noise stimulation. J. Neurosci., 28: 14147-55, 2008.

14- MINHAS P., BANSAL V., PATEL J., HO J., DIAZ J., DATTA A. and BIKSON M.: Electrodes for highdefinition transcutaneous DC stimulation for applications in drug delivery and electrotherapy, including tDCS. J. Neurosci. Methods Jul., 15, (190(2)), 2010.

15- SOLER M.D., KUMRU H., PELAYO R., VIDAL J., TORMOS J.M., et al.: Effectiveness of transcranial direct current stimulation and visual illusion on neuropathic pain in spinal cord injury. Brain, 133: 2565-77, 2010.

16- VALLE A., ROIZENBLATT S., BOTTE S., ZAGHI S., RIBERTO M. and TUFIK S.: Efficacy of anodal transcra- nial Direct Current Stimulation (tDCS) for the treatment of fibromyalgia: Results of a randomized, sham-controlled longitudinal clinical trial. J. Pain Manag., 2 (3): 353-61, 2009.

17- VASEGHI B., ZOGHI M. and JABERZADEH S.: Does anodal transcranial direct current stimulation modulate sensory perception and pain? A meta-analysis study. Clin Neurophysiol. 125: 1847-58. 10.1016/j . clinph.2014. 01.020 .

18- O'CONNELL N.E., WAND B.M., MARSTON L., SPENCER S. and DeSOUZA L.H.: Stimulating the brain without surgery in the management of chronic pain. Non-invasive brain stimulation techniques for chronic pain. Cochrane Database of Systematic Reviews, Issue 4. Art. No.: CD008208. Doi: 10.1002/14651 858.CD008208.pub3, 2014.

19- GOSSRAU G., WAHNER M., KUSCHKE M., KONRAD B., REICHMANN H., WIEDEMANN B., et al.: Microcurrent transcutaneous electric nerve stimulation in painful diabetic neuropathy: A randomized placebocontrolled study. Pain Med., 12 (6): 953-60. doi: 10.1111/j.15264637.2011.01140.x, 2011.

20- JIN D.M., XU Y., GENG D.F. and YAN T.B.: Effect of transcutaneous electrical nerve stimulation on symptomatic diabetic peripheral neuropathy: A meta-analysis of randomized controlled trials. Diabetes Res. Clin. Pract., 89 (1): 10-5. doi: 10.1016/j.diabres.2010.03.021, 2010.

21- DUBINSKY R.M. and MIYASAKI J.: Assessment: Efficacy of transcutaneous electric nerve stimulation in the treatment of pain in neurologic disorders (as evidence based review): Report of the Therapeutics and Technology Assessment Subcommittee of the American Academy of Neurology. Neurology, 74: 173-6, 2010.

22- STEIN C., EIBEL B., SBRUZZI G., LAGO P.D. and PLENTZ R.D.M.: Electrical stimulation and electromagnetic field use in patients with diabetic neuropathy: Systematic review and meta-analysis. Braz. J. Ther., 17 (2), pp. 93-104, 2013.

23- DONNELAN C.P. and CALDWELL K.: TE NS and FES for sensory impairment and gait dysfunction following removal of spinal cord ependymoma. Phys. Res. Int., 14: 234-41, 2009.

24- LAUFER Y. and ELBOIM-GABYZON M.: Does sensory transcutaneous electrical stimulation enhance motor recovery following a stroke: A systematic review. Neurorehabil. Neurol. Repair., 25: 799-809, 2011.

25- CELIK E.C., ERHAN B., GUNDUZ B. and LAKSE E.: The effect of lowfrequency TENS in the treatment of neuropathic pain in patients with spinal cord injury. Spinal Cord., 51 (4): 334-7, 2013.

26- NORRBRINK C.: Transcutaneous electrical nerve stimulation for treatment of spinal cord injury neuropathic pain. J. Rehabil. Res. Dev., 46: 85-94, 2009.

27- OOSTERHOF J., SAMWEL H.J., De BOO T.M., et al.: Predicting outcome of TENS in chronic pain: A prospective, randomized, placebo controlled trial. Pain, 136: 11$20,2008$. 


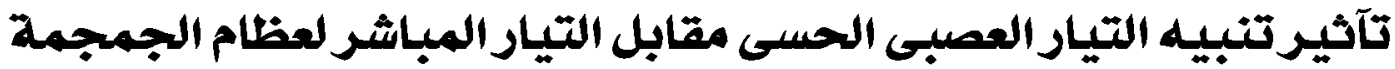

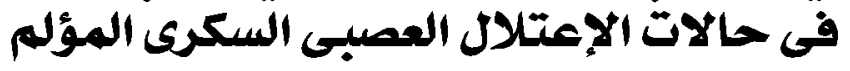

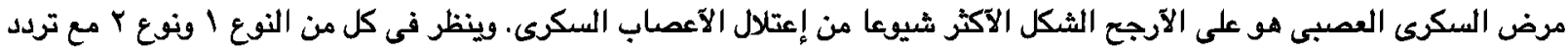

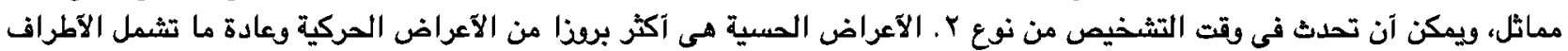

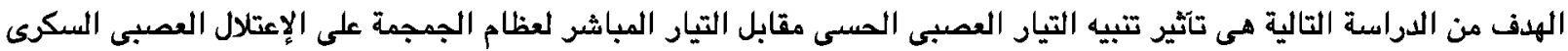

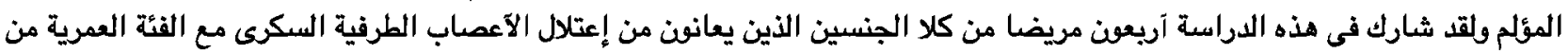

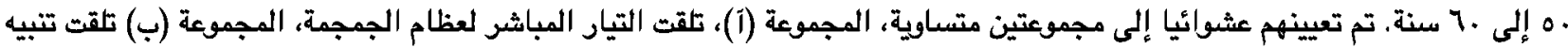

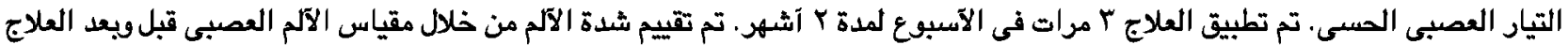

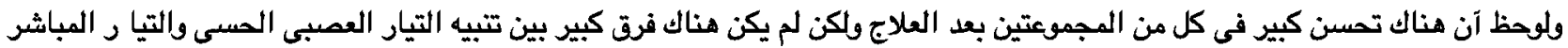
لعظام الجمجمة. الإستتاج:كلا من تنبيه التيار العصبى الحسى والتيارالمباشر لعظام الجمجمة على حد سواء فعالة فى الآلم المرتبطة إعتلال الآعصاب المئلم السكرى. 